\title{
Monocyte Chemotactic and Activating Factor Is a Potent Histamine-releasing Factor for Basophils
}

Rafeul Alam, Michael A. Lett-Brown, Patricia A. Forsythe, Diedra J. Anderson-Walters, Claire Kenamore, Csilla Kormos, and J. Andrew Grant

University of Texas Medical Branch, Department of Internal Medicine, Division of Allergy and Immunology, Galveston, Texas 77550

\begin{abstract}
Monocyte chemotactic and activating factor (MCAF) is a recently cloned cytokine that causes chemotaxis of basophils. In our pursuit of cytokines affecting basophil function, we studied the effect of MCAF on histamine secretion from basophils. Leukocytes from 20 donors, 10 allergic and 10 normal subjects, were studied. MCAF caused dose-dependent release of histamine at concentrations of $10^{-8}$ and $10^{-7} \mathrm{M}$, and the mean release was $31.25 \pm 2.9 \%$ at the highest concentration. In the same experiments the mean histamine release by anti-IgE and histamine releasing factor (HRF) was $27.05 \pm 4 \%$ and $32.70 \pm 2.7 \%$, respectively. All 20 subjects responded to MCAF with significant histamine release. Allergic subjects released significantly more histamine than normals in response to anti-IgE $(P<0.01)$ but not to MCAF $(P=0.2)$ and HRF $(P=0.1)$. The histamine release was significantly correlated between MCAF and HRF $(P<0.01)$, but not between MCAF and anti-IgE $(P>0.05)$. The histamine release by MCAF was complete within the first 3 min. MCAF-induced degranulation was a calcium-dependent process. Leukocytes depleted of monocytes responded equally well to MCAF. Using an anti-MCAF affinity column we determined that $>50 \%$ of HRF activity of crude PBMC supernatant could be attributed to MCAF. Thus, we established that MCAF is a potent secretagogue for basophils. ( J. Clin. Invest. 1992. 89:723-728.) Key words: cytokine $\bullet$ monocyte chemoattractant protein-1 $\bullet$ mediator $\bullet$ allergy
\end{abstract}

\section{Introduction}

Chronic allergy is characterized by infiltration of the tissue with various inflammatory cells, including eosinophils, mast cells, basophils, lymphocytes, and neutrophils. This allergic inflammation is reminiscent of the late phase reaction to allergen challenge under experimental conditions. A growing body of

Address correspondence to Rafeul Alam, M. D., Ph. D., Division of Allergy and Immunology, Department of Internal Medicine, University of Texas Medical Branch, G-62, Galveston, TX 77550.

Received for publication 1 July 1991 and in revised form 9 October 1991.

1. Abbreviations used in this paper: GM-CSF, granulocyte macrophage-colony-stimulating factor; HA, Hepes-buffered saline, $\mathrm{pH} 7.4$ and $0.03 \%$ HSA; HACM, Hepes-buffered saline, pH 7.4, 0.03\% HSA, 2 $\mathrm{mM} \mathrm{CaCl}_{2}$, and $1 \mathrm{mM} \mathrm{MgCl} ; \mathrm{HCM}$, Hepes-buffered saline with $\mathrm{CaCl}_{2}$ and $\mathrm{MgCl}_{2} ; \mathrm{HRF}$, histamine-releasing factor; MCAF, monocyte chemotactic and activating factor.

J. Clin. Invest.

(C) The American Society for Clinical Investigation, Inc.

0021-9738/92/03/0723/06 \$2.00

Volume 89, March 1992, 723-728 evidence suggests that cytokines may play a crucial role in the recruitment and activation of inflammatory cells. Increased expression of various cytokines has been demonstrated in tissue specimens from late phase allergic reactions (1). Cytokines that activate basophils include IL-3, granulocyte macrophagecolony-stimulating factor (GM-CSF) and connective tissue activating peptide III/neutrophil activating peptide-2 $(2,3)$. Some less defined histamine-releasing factors apparently distinct from most known cytokines have been described and are being characterized. One form of histamine releasing factor (HRF) has been previously found to coelute from various chromatographic columns with basophil chemotactic factor (4). Also, monocyte chemotactic factor showed a similar chromatographic elution profile as did basophil chemotactic factor (5). Therefore, we decided to study whether the recently cloned monocyte chemotactic and activating factor (MCAF)/monocyte chemoattractant protein-1 $(6,7)$ releases histamine from basophils. We also compared the activity of MCAF with HRF and anti-IgE antibody.

\section{Materials and Methods}

Reagents. RPMI 1640 was obtained from Gibco Laboratories, Grand Island, NY; HSA, glutamine, Histopaque-1077, Con A, penicillin, streptomycin, FMLP, and C5a from Sigma Chemical Co., St. Louis, MO; Hepes from Research Organics, Inc., Cleveland, OH; hydroxyethyl starch (HetaStarch) from American McGaw, Irvine, CA; MCAF was from PeproTech Inc., Rocky Hills, NJ. The material was $99 \%$ pure by SDS-PAGE and reverse phase HPLC. It was received as a lyophilized material in PBS. A stock solution of MCAF was made at a concentration of $2 \times 10^{-6} \mathrm{M}$ in Hepes-buffered saline, $\mathrm{pH} 7.4$ containing $0.3 \% \mathrm{HSA}, 2 \mathrm{mM} \mathrm{CaCl}{ }_{2}$, and $1 \mathrm{mM} \mathrm{MgCl}_{2}$. Affinity-purified rabbit anti-human IgE serum $(1 \mathrm{mg} / \mathrm{ml})$ was from Behring Diagnostics, Somerville, NY. Hunter's Titermax was obtained from CytRx Corp., Norcross, GA.

Generation of HRF-containing supernatant. Leukocytes were isolated from buffy coats obtained from normal blood bank donors. PBMC were isolated by Ficoll-Hypaque gradient centrifugation as previously described (8) and pulsed with Con A $25 \mu \mathrm{g} / \mathrm{ml}$ in RPMI 1640 medium for $12 \mathrm{~h}$, resuspended in RPMI 1640 medium, and then cultured for $72 \mathrm{~h}$. Supernatants were harvested and concentrated $50 \times$ using an ultrafiltration chamber with YM-5 filters (mol wt cutoff 5,000; Amicon, Beverly, MA) and ultracentrifuged. Samples were aliquoted, frozen at $-70^{\circ} \mathrm{C}$, and used as a source of HRF. Samples of $\mathrm{HRF}$ were preincubated with $0.02 \mathrm{M}$ alpha-methyl mannoside for 30 min before use. The concentration of protein in this preparation was 8 $\mu \mathrm{g} / \mathrm{ml}$.

Isolation of peripheral blood leukocytes. Donors for this study were selected from a large group of allergic and nonallergic subjects that were screened in our laboratory for histamine release from basophils in response to HRF and anti-IgE. Allergic status was defined by the presence of clinical symptoms, past allergic history, and positive reaction to prick skin testing with a panel of 32 local aeroallergens.

Venous blood from donors was anticoagulated with $10 \mathrm{mM}$ EDTA and sedimented with $1.5 \%$ hydroxyethyl starch for $30 \mathrm{~min}$ at room 
temperature (8). The leukocyte-rich buffy coat was collected and washed three times in HA buffer (Hepes-buffered saline, $\mathrm{pH} 7.4$ and $0.03 \% \mathrm{HSA})$ in a refrigerated centrifuge $\left(4^{\circ} \mathrm{C}\right)$ at $300 \mathrm{~g}$. The washed leukocytes were suspended in HACM buffer (Hepes-buffered saline, pH 7.4, 0.03\% HSA, $2 \mathrm{mM} \mathrm{CaCl}_{2}$, and $1 \mathrm{mM} \mathrm{MgCl}_{2}$ ). Leukocytes from $1 \mathrm{ml}$ of blood were usually used for one duplicate experiment.

In some experiments mononuclear cells containing 1-2\% basophils were purified by Ficoll-Hypaque gradient centrifugation and further processed for use in the histamine release test. To study the effect of depletion of monocytes on MCAF-induced histamine release from basophils, mononuclear cells were suspended in RPMI 1640 with $0.1 \%$ $\mathrm{HSA}$ and incubated on plastic petri dishes for $1 \mathrm{~h}$ at $37^{\circ} \mathrm{C}$ as described previously (8). Nonadhered cells were collected, washed, and used in the histamine release test. The cells were stained with OKM1 antibody followed by FITC-labelled goat anti-mouse IgG to check the percentage of monocytes. The contamination with monocytes was $<2 \%$. In parallel experiments mononuclear cells that were incubated in the same medium for $1 \mathrm{~h}$ in a polypropylene tube were used as a control (10-20\% monocytes). The concentration of basophils in both preparations was $\sim 1 \%$.

Histamine release assay. Aliquots of $50 \mu \mathrm{l}$ of various dilutions of MCAF, HRF, or anti-IgE antibody were incubated with $50 \mu$ l of leukocyte suspension for $45 \mathrm{~min}$ in a shaking water bath at $37^{\circ} \mathrm{C}$. Each experiment was done in duplicate. $400 \mu \mathrm{l}$ of HA buffer was added to each tube at the end of the incubation. The supernatants were separated from the cells by centrifugation at $600 \mathrm{~g}$ for $5 \mathrm{~min}$ at $4^{\circ} \mathrm{C}$. The histamine content of the supernatants was measured using an automated fluorometric analyzer (8). Spontaneous histamine release was assessed by incubating the cells in HACM buffer alone. The total histamine content of the cells was measured by lysing the cells with 3\% perchloric acid. The percentage of histamine release was calculated according to the formula: ([histamine in the supernatant] $\times 100) /($ total histamine in the cells). Spontaneous histamine release from the cells was usually $<5 \%$. The values of spontaneous histamine release were subtracted from the calculated histamine release.

Treatment of basophils with lactic acid and resensitization with serum. The removal of surface IgE from basophils was accomplished by treating the cells with lactic acid (pH 3.9) for 3.5 min according to Pruzansky et al. (9). In control experiments, the cells were incubated in HA buffer. The cells were then washed three times and resensitized for 45 min with serum from allergic asthmatic patients or healthy subjects. After two more washes, the cells were used in the histamine release test.

Desensitization experiment. All secretagogues used in these experiments were prepared in calcium- and magnesium-free HA buffer. A sample of HRF was dialyzed against calcium- and magnesium-free Hepes-buffered saline and then preincubated with 4 mM EDTA before use. Leukocytes were washed in calcium- and magnesium-free HA in the presence of $4 \mathrm{mM}$ EDTA and then suspended in calcium- and magnesium-free HA buffer. Aliquots of leukocytes were preincubated with MCAF, HRF, and anti-IgE for $45 \mathrm{~min}$. The cells were then washed $3 \times$, suspended in HACM, and then challenged with MCAF, HRF, and anti-IgE (all prepared in calcium- and magnesium-containing buffers). The incubation was continued for another $45 \mathrm{~min}$. The released histamine in the supernatant was measured.

Affinity chromatography. A rabbit was immunized with MCAF in Titermax. Polyclonal antibodies were detected after four injections. The IgG fraction was separated on a protein A-agarose column. An immunoaffinity column was prepared using $\mathrm{CNBr}$-activated Sepharose according to manufacturer's instructions. A sample of MCAF or crude HRF supernatant was applied to the immunoaffinity column. The column was washed with 4-bed vol of Hepes-buffered saline with $\mathrm{CaCl}_{2}$ and $\mathrm{MgCl}_{2}(\mathrm{HCM})$ buffer, and then eluted with a gradient of 0-6 $\mathrm{M} \mathrm{KSCN}$. The samples were dialyzed against HCM and then assayed for HRF activity.

Results were expressed as mean \pm SEM. Data were analyzed for statistical significance using appropriate statistical tests as indicated in the text.

\section{Results}

Leukocytes from 20 subjects were studied for histamine release by MCAF, anti-IgE, and HRF. The concentration of MCAF was chosen on the basis of published data on monocyte activation (optimal $10^{-9}$ to $10^{-8} \mathrm{M}$, ref. 10). All donors were previously screened for release to anti-IgE and HRF. The optimal concentration of anti-IgE for inducing histamine release was a 1:1,000 dilution of the antibody. The concentration of HRF was arbitrary, although the protein concentration of the HRF preparation $(8 \mu \mathrm{g} / \mathrm{ml})$ was used as the guideline. Basophils from all 20 subjects responded to MCAF, and the mean histamine release was $31.35 \pm 2.9 \%$. By comparison the mean histamine release by anti-IgE and $\mathrm{HRF}$ was $27.05 \pm 4 \%$ and $32.70 \pm 2.7 \%$, respectively, and the differences among the means were not statistically significant $(P>0.05$, analysis of variance). The dose-response curve for each secretagogue is shown in Fig. 1. With basophils from two donors an extended dose-response experiment with MCAF up to $10^{-6} \mathrm{M}$ resulted only in a small increase in histamine release $\left(34 \pm 3 \%\right.$ at $10^{-7} \mathrm{M}$ vs $37 \pm 3 \%$ at $\left.10^{-6} \mathrm{M}\right)$. Since basophils from allergic subjects have been shown to have increased releasability, the results were analyzed on the basis of allergic phenotype of the donors. Among the 20 donors, 10 were allergic as assessed by the positive skin response to common aeroallergens and the presence of clinical
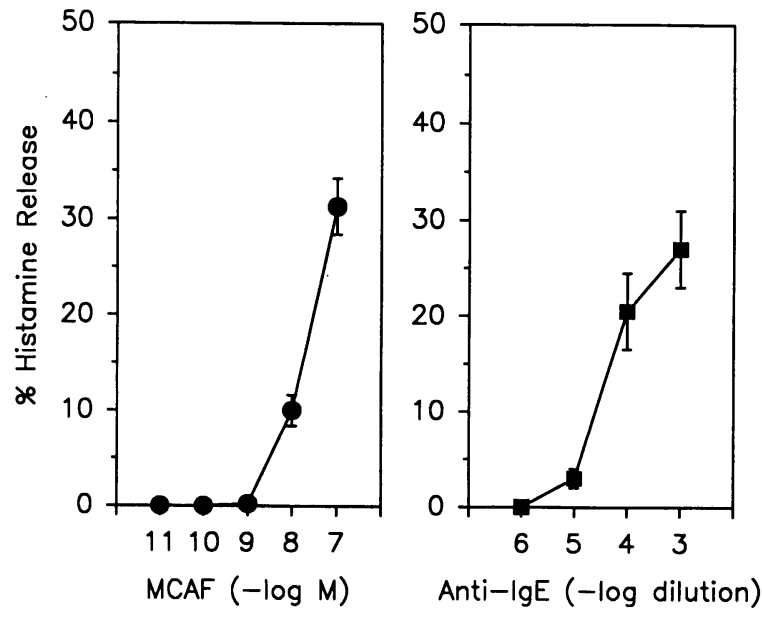

Figure 1. Dose-response curves for MCAF-, antiIgE-, and HRF-induced histamine release from basophils. Leukocytes from 20 donors were incubated with various concentrations of MCAF, antiIgE, and HRF, and the histamine release in the supernatant was measured spectrofluorometrically. Results are expressed as percent intracellular release (mean \pm SEM). 


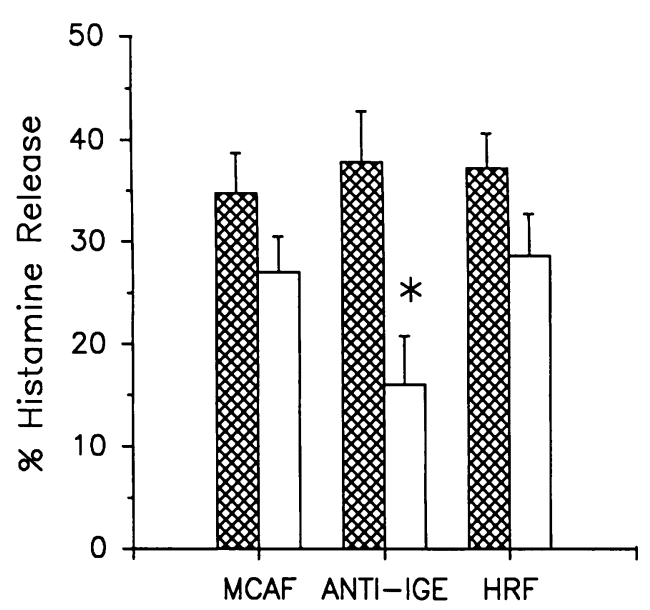

Figure 2. Comparison of histamine release from basophils between allergic and nonallergic subjects ( $n=10$ in each group) in response to MCAF $\left(10^{-7} \mathrm{M}\right)$, anti-IgE (1:1,000 dilution), and HRF (undiluted). *Statistical significance at $P=0.01$. Allergic; $\square$, normal.

symptoms of rhinitis and asthma. The other 10 donors were normal by the same criteria. The mean percentage of histamine released by MCAF, anti-IgE, and HRF was $34.7 \pm 4.1 \%$, $37.8 \pm 4.8 \%$, and $37.2 \pm 3.5 \%$, respectively, in the allergic group, and $27.0 \pm 3.5 \%, 16 \pm 4.8 \%$, and $28.6 \pm 4.1 \%$, respectively, in the normal group (Fig. 2). The difference in IgE-induced release was statistically significant between the two groups $(P=0.01$, Wilcoxon's rank sum test) but the difference was not significant for MCAF and HRF although both showed a tendency toward a higher release in the allergic group $(P=0.2$ for MCAF, $P=0.1$ for HRF). There was a significant correlation of histamine release between MCAF and HRF (Fig. 3, $A$ and $B)$. The correlation coefficient ( $r$ ) was 0.73 at the lower concentration, and 0.56 at the higher concentration of MCAF and HRF by Spearman's rank correlation $(P<0.01)$. No such correlation was found between MCAF and anti-IgE $(r=0.19$ or lower at all concentrations analyzed, $P>0.05$ ).

The kinetics of histamine release by MCAF are shown in Fig. 4. The release was very rapid, and $>80 \%$ of the maximal release took place within $30 \mathrm{~s}$. The release was almost complete within the first $3 \mathrm{~min}$. HRF showed a similar kinetics profile. On the other hand, IgE consistently required a lag period of 1-3 min before any noticeable release occurred. To investigate whether the degranulation of basophils by MCAF is a calciumdependent phenomenon, we conducted the experiments in Hepes-albumin buffer in the presence of $4 \mathrm{mM}$ EDTA containing no calcium or magnesium. In parallel experiments leukocytes were incubated with MCAF in the calcium containing HACM buffer. The release of histamine was 0 and $43 \pm 3 \%$ in the absence or presence of calcium and magnesium, respectively (three duplicate experiments). As anticipated, a similar lack of histamine release was observed with anti-IgE and HRF in parallel experiments (data not shown).

Monocytes have previously been shown to produce HRF (8). Since MCAF is an activator of monocytes, it is possible that HRF was released during the incubation and caused histamine release. To address this issue we conducted experiments with mononuclear cell preparations depleted of monocytes. Parallel experiments were carried out with cells containing monocytes. As shown in Fig. 5, the depletion of monocytes did not affect the histamine release from basophils.

Some forms of HRF have previously been shown to be IgE-dependent $(11,12)$. This conclusion was drawn on the basis of the loss of response of basophils upon IgE-stripping with lactic acid. We have conducted similar experiments with MCAF. The IgE-stripped basophils showed a significant reduction of histamine release to anti-IgE as well as to HRF and MCAF (Fig. 6). The response to anti-IgE was restored by both allergic and normal serum. The response to MCAF was restored to a certain degree by sensitization of the basophils with serum from two of three allergic patients and two of three normal subjects. The response to HRF was partially restored by serum from most donors. The release by FMLP was not affected by the stripping procedure.

Desensitization experiments demonstrated that MCAF desensitized to itself completely within $10 \mathrm{~min}$. A significant selfdesensitization was also observed with anti-IgE and HRF, but required a longer preincubation, for $45 \mathrm{~min}$, and the desensitization was not complete. Preincubation of cells with anti-IgE caused a small but significant cross-desensitization to HRF and MCAF (Fig. 7). However, the reverse was not true. On the
A



MCAF $10^{-7} \mathrm{M}$
$B$

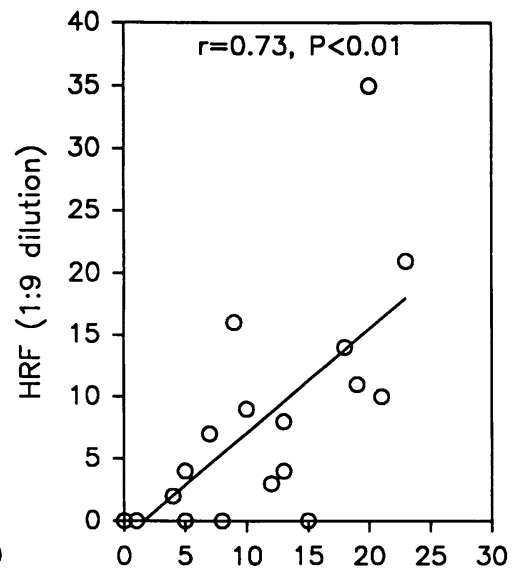

MCAF $10^{-8} \mathrm{M}$
Figure 3. Correlation of basophil histamine release between HRF and MCAF. Leukocytes from 20 donors were incubated with HRF (1:9 and undiluted) and MCAF $\left(10^{-8}\right.$ and $\left.10^{-7} \mathrm{M}\right)$, and the histamine release was measured. Results are expressed as mean histamine release by each agonist. The correlation of histamine release at the lower concentration $\left(10^{-8} \mathrm{M}\right.$ of MCAF vs. 1:9 dilution of HRF, $A)$ and higher concentration $\left(10^{-7} \mathrm{M}\right.$ of MCAF vs. undiluted HRF, $B$ ) of the secretagogues is shown. 


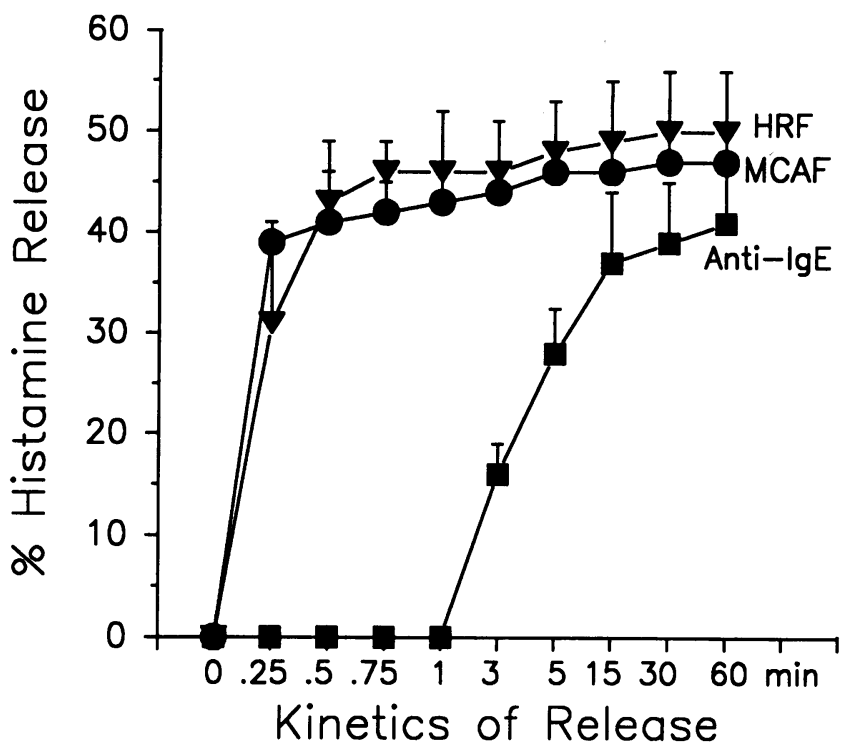

Figure 4. The kinetics of MCAF-, anti-IgE- and HRF-induced histamine release from leukocytes. Leukocytes from four different donors were incubated with $\operatorname{MCAF}\left(10^{-7} \mathrm{M}\right)$, anti-IgE (1:1,000 dilution), and HRF (undiluted) for different periods of time as indicated. At the conclusion ice-cold buffer containing $4 \mathrm{mM}$ EDTA was added, and the supernatant was separated by centrifugation at $4^{\circ} \mathrm{C}$. Results of four duplicate experiments are shown.

other hand, there was a partial cross-desensitization between $\mathrm{MCAF}$ and $\mathrm{HRF}$.

The contribution of MCAF to the histamine-releasing activity of the crude PBMC supernatant was determined by passing the latter through an anti-MCAF affinity column. The affinity column was prepared using purified rabbit polyclonal IgG antibody raised against MCAF. A little more than half of the activity was bound to the column and was then eluted with $6 \mathrm{M}$ KSCN (Fig. 8). In parallel experiments, the binding of MCAF to the affinity column was $100 \%$. Similar results were obtained using polyclonal antibodies against MCAF obtained from Peprotech.

\section{Discussion}

We have demonstrated that MCAF is a potent histamine releasing factor. At the doses used in this study, its histamine releasing capability is comparable to anti-IgE and HRF. More importantly, every single donor that we have tested, thus far, responded to MCAF with a significant histamine release. Allergic patients showed a tendency to release more histamine in response to MCAF and HRF than normals but the results did not reach statistical significance perhaps due to the low number of patients. This is the first report of MCAF causing histamine release from basophils. Most cytokines have pleiotropic activity. Our report points to the pleiotropism of another cytokine, namely MCAF.

HRF represents a heterogeneous group of cytokines, the common denominator being the direct histamine release from basophils. Baeza et al. (13) and our group (14) have shown that there are at least three species of HRF in the molecular weight range 8-12, 17-20, and 40-45 kD. Recently, connective tissue activating peptide III and its cleavage product neutrophil activating peptide- 2 have been identified as the low molecular weight species of $\mathrm{HRF}$ (3). Both of them release histamine from basophils. As mentioned earlier, IL-3 and GM-CSF cause histamine release from some selected allergic patients $(2,15,16)$. All of our 20 cell donors were previously tested with IL-3 and GM-CSF. Only one donor released histamine in response to IL-3 and GM-CSF at micromolar concentrations. Thus, MCAF appears to be one of the most potent chemically defined histamine releasing cytokines.

$\mathrm{MCAF}$ is an $8.7-\mathrm{kD}$ protein based on amino acid sequence and cloning data (10) although it migrates as a $13-15-\mathrm{kD}$ protein on SDS-PAGE. MCAF belongs to the family of cytokines called intercrine-beta that have four conserved cysteine residues. The first two cystine residues are adjacent. The other members of this family include hRANTES, hAct-2h, mJE, mTCA, mMIP-1 alpha and beta (reviewed in ref. 10). Analysis of the human genomic DNA suggests the presence of a single gene for MCAF. MCAF is produced by human lymphocytes, fibroblasts, endothelial cells, baboon arterial smooth muscle, and a number of tumor cell lines.

MCAF is a potent chemotactic factor for monocytes at a concentration of $10^{-9}$ to $10^{-8} \mathrm{M}$. At higher concentrations it also activates them to cause tumor cell killing, $N$-acetyl betaglucosaminidase and superoxide anion release (17). MCAF has no activity on neutrophils and eosinophils. However, it is chemotactic for basophils (10). Since most chemotactic factors also possess secretagogue activity, our finding on histamine releasing activity of MCAF reinforces this notion. The optimal concentration of MCAF for release of histamine $\left(10^{-7} \mathrm{M}\right)$ was higher than that published for chemotaxis of monocytes in the literature. We verified the activity of our MCAF preparation, and found that the optimal concentration for monocyte chemotaxis was $10^{-9} \mathrm{M}$. As mentioned earlier, the activation of monocytes for superoxide generation was found to require

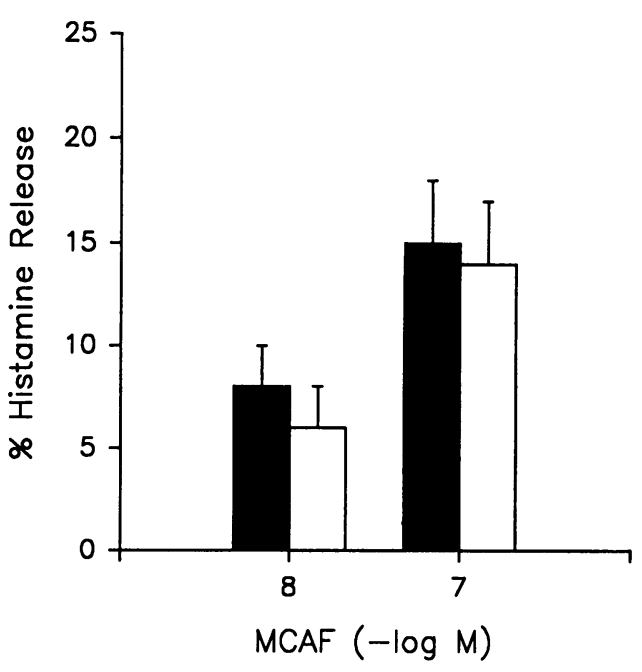

Figure 5. The effect of depletion of monocytes on MCAF-induced histamine release from leukocytes. Ficoll-Hypaque-purified mononuclear cells were depleted of monocytes by plastic adherence and then tested in the release experiments. In parallel experiments cell preparations containing monocytes were used. The basophil content of the preparations was $\sim 1 \%$. The results of three duplicate experiments are shown. There was no difference in histamine release between monocyte-containing and monocyte-depleted preparations $(P$ $>0.05$, Wilcoxon's rank sum test). $m$, Monocyte-rich; $\square$, monocytedepleted. 


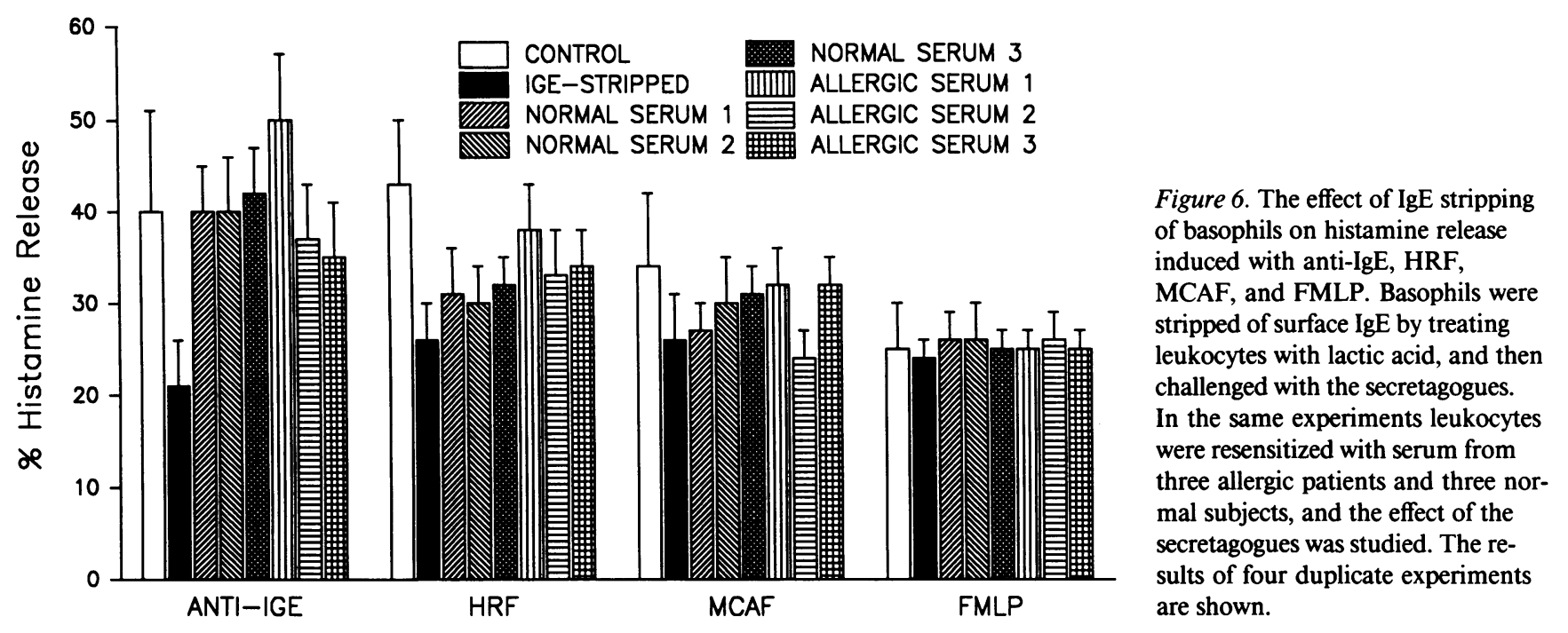

concentrations of MCAF higher than that needed for chemotaxis (17). Similar results were observed with other agonists such as FMLP (18).

The histamine release experiments with MCAF were conducted with leukocytes containing $0.5-2 \%$ basophils. Monocytes have previously been shown to produce HRF (6). Since MCAF is an activator of monocytes, it is possible that the release of histamine by MCAF is secondary to monocyte-derived HRF. However, we think this unlikely. The action of MCAF is very rapid, and the release is complete within three minutes. Furthermore, the depletion of monocytes did not affect histamine release by MCAF. However, a definitive answer will require experiments using a homogeneous population of purified basophils.

Some species of HRF have been shown to be IgE dependent. The IgE dependency has been defined as the lack of response of IgE-stripped basophils to HRF and the restoration of the response by serum from some allergic patients only. Similar IgE dependency has been demonstrated for IL-3 and $\mathrm{D}_{2} \mathrm{O}(16$, 19). Using the same protocol of lactic acid treatment of basophils we found that the response of basophils to MCAF decreased significantly upon IgE stripping, although the decrease was not as profound as with anti-IgE and HRF. The response was restored with serum from some allergic as well as normal subjects. Similar results were obtained with $\mathrm{HRF}$ and anti-IgE. Although the results of IgE-stripping experiments might suggest some IgE dependency of MCAF (and HRF), the results of kinetics experiments, the complete lack of correlation of release between MCAF and anti-IgE, and the good response of normal basophils to MCAF, go against this notion. Although MCAF and PBMC-derived HRF are active on normal basophils, in agreement with MacDonald et al. (11) we previously reported that bronchoalveolar lavage-derived HRF was not as active with normal basophils as with basophils from allergic subjects.

The results of desensitization experiments were not conclusive. Although anti-IgE partially cross-desensitized MCAF and HRF, the reverse was not true. One possible explanation of these findings would be that both anti-IgE and MCAF have independent intracellular secretory pathways. However, the IgE-mediated secretory pathway may influence MCAF but not vice versa. The lactic acid treatment in the stripping experiment may affect, in addition to IgE, the receptors for some secretagogues, e.g., MCAF, but not FMLP.

Our experiment with the immunoaffinity column established that MCAF significantly contributed to the histamine



Pretreated with anti-lgE

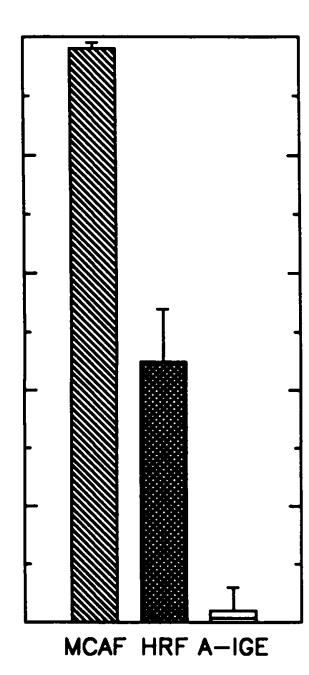

Pretreated with MCAF

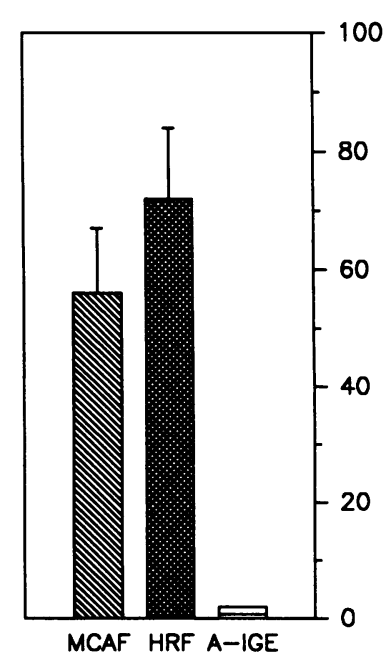

Pretreated with HRF
Figure 7. Cross-desensitization of anti-IgE (A-IgE), MCAF and HRF. Leukocytes were preincubated with anti-IgE, MCAF and HRF in calcium- and magnesium-free $\mathrm{HA}$ buffer for $45 \mathrm{~min}$. The cells were then washed, suspended in HACM and challenged with the three secretagogues. At each step of the experiment a control was run with the buffer which was always negative. The results of four duplicate experiments are shown. 


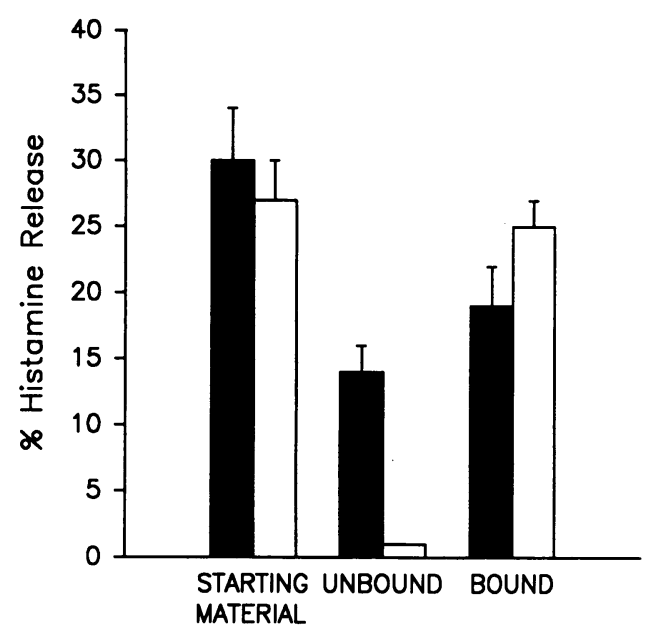

Figure 8. Affinity chromatography of crude PBMC supernatant and MCAF. A sample of HRF or MCAF was passed through an affinity column containing anti-MCAF rabbit polyclonal antibodies. The column was washed with 4-bed vol of Hepes-buffered saline with $\mathrm{CaCl}_{2}$ and $\mathrm{MgCl}_{2}$ and then eluted with a gradient of 0-6 M KSCN. The dialyzed fractions were tested for histamine releasing activity. Results of three experiments are shown. $n$, HRF; $\square$, MCAF.

releasing activity of the crude PBMC supernatant. There was a significant correlation between the release by MCAF and HRF. Also, the kinetics of release by MCAF and HRF were similar. However, a significant proportion of HRF did not bind to the affinity column. MCAF has been identified in several molecular weight forms $(10,13$, and $15 \mathrm{kD})$ perhaps due to posttranslational modifications. There has been no report of the existence of multimeric forms of MCAF that would resemble the 41-kD HRF species. It is possible that the molecular identity of some species of HRF remains to be determined.

MCAF may play an important role in the pathogenesis of allergic inflammation. Although the effect of MCAF on human mast cells is not yet known, basophils appear to play a significant role in the pathogenesis of late phase allergic reactions. The mediator release profile indicates the presence of histamine but not $\mathrm{PGD}_{2}$ in the late phase reaction (20). Mast cells produce both histamine and $\mathrm{PGD}_{2}$, while basophils synthesize histamine but not $\mathrm{PGD}_{2}$ (20). The latter observation points to the participation of basophils but not mast cells in the late allergic reaction. HRF-like molecules have been detected in the blister fluid from late phase cutaneous allergic reactions (21), bronchoalveolar lavage fluid (22), and nasal washings (8). Increased synthesis of HRF by mononuclear cells has been reported in asthma (23) and atopic dermatitis with food allergy (24). Availability of an ELISA for MCAF in the near future will help establish the validity of the foregoing observations, and delineate the importance of this cytokine in allergic and other chronic inflammatory diseases.

\section{Acknowledgments}

This work was supported by a Burroughs Wellcome DIA Merit Award, a John Sealy Award, and National Institute of Allergy and Infectious Diseases grants AI27864 and AI22940.

\section{References}

1. Kay, A. B., S. Ying, V. Varney, M. Gaga, S. R. Durham, R. Moqbel, A. J. Wardlaw, and Q. Hamid. 1991. Messenger RNA expression of the cytokine gene cluster, interleukin 3 (IL-3), IL-4, IL-5 and granulocyte/macrophage colony stim- ulating factor in allergen-induced late phase cutaneous reactions in atopic subjects. J. Exp. Med. 173:775-778.

2. Alam, R., J. B. Welter, P. A. Forsythe, M. A. Lett-Brown, and J. A. Grant 1989. Comparative effect of recombinant IL-1, $-2,-3,-4$, and -6 , IFN-gamma granulocyte-macrophage colony stimulating factor, tumor necrosis factor-alpha, and histamine-releasing factors on the secretion of histamine release from basophils. J. Immunol. 142:3431-3435.

3. Baeza, M. L., S. R. Reddigari, D. Kornfeld, N. Ramani, E. M. Smith, P. A. Hossler, T. Fischer, C. W. Castor, P. G. Gorevic, and A. P. Kaplan. 1990. Relationship of one form of human histamine-releasing factor to connective tissue activating peptide-III. J. Clin. Invest. 85:1516-1521.

4. Thueson, D. O., D. E. Plank, M. A. Lett-Brown, and J. A. Grant. 1982. Lymphokine-induced release of basophilic histamine: separation of the activity into two factors. In Human Lymphokines. A. Khan and D. Hills, editors. Academic Press, New York. 229-239.

5. Lett-Brown, M. A., D. A. Boecher, and E. J. Leonard. 1976. Chemotactic response of normal human basophils to $\mathrm{C} 5 \mathrm{a}$ and to lymphocyte-derived chemotactic factor. J. Immunol. 117:246-252.

6. Yoshimura, T., N. Yuhki, S. K. Moore, E. Appella, M. I. Lerman, and E. J. Leonard. 1989. Human monocyte chemoattractant protein-1 (MCP-1). Fulllength cDNA cloning, expression in mitogen-stimulated blood mononuclear leukocytes, and sequence similarity to mouse competence gene JE. FEBS(Fed. Eur. Biochem. Soc.) Lett. 244:487-493.

7. Furutani, Y., H. Nomura, M. Notake, Y. Oyamad, T. Fukui, M. Yamada, C. G. Larsen, J. O. Oppenheim, and K. Matsushima. 1989. Cloning and sequencing of the cDNA for human monocyte chemotactic and activating factor (MCAF). Biochem. Biophys. Res. Commun. 159:249-255.

8. Alam, R., P. A. Forsythe, M. A. Lett-Brown, and J. A. Grant. 1989. Cellular origin of histamine-releasing factor produced by peripheral blood mononuclear cells. J. Immunol. 142:3951-3956.

9. Pruzansky, J. J., L. C. Grammer, R. Patterson, and M. Roberts. 1983. Dissociation of IgE from receptors on human basophils. I. Enhanced passive sensitization for histamine release. J. Immunol. 131:1949-1953.

10. Leonard, E. J., and T. Yoshimura. 1990. Human monocyte chemoattractant protein-1 (MCP-1). Immunol. Today. 11:97-101.

11. MacDonald, S. M., L. M. Lichtenstein, D. Proud, M. Plaut, R. M. Naclerio, D. W. MacGlashan, and A. Kagey-Sobotka. 1987. Studies of IgE-dependent histamine releasing factors: heterogeneity of IgE. J. Immunol. 139:506-512.

12. Alam, R., P. A. Forsythe, J. A. Rankin, M. C. Boyars, M. A. Lett-Brown, and J. A. Grant. 1990. Sensitivity of basophils to histamine releasing factors of various origin: dependency on allergic phenotype of the donor and surface-bound IgE. J. Allergy Clin. Immunol. 86:73-81.

13. Alam, R., P. A. Forsythe, J. Welter, M. A. Lett-Brown, and J. A. Grant. 1990. Purification of histamine releasing factor (HRF) produced by mononuclear cells and cell lines. J. Allergy Clin. Immunol. 85:257. (Abstr.)

14. Baeza, M. L., S. R. Reddigari, M. Haak-Frendscho, D. Kornfeld, P. G. Gorevic, and A. P. Kaplan. 1988. Purification and characterization of human mononuclear cell histamine-releasing factor. J. Clin. Invest. 83:1204-1210.

15. Haak-Frendscho, M., N. Arai, K.-I. Arai, M. L. Baeza, A. Finn, and A. P. Kaplan. 1988. Human recombinant granulocyte-macrophage colony stimulating factor and interleukin 3 cause basophil histamine release. J. Clin. Invest. 82:1720.

16. MacDonald, S., R. P. Schleimer, A. Kagey-Sobotka, S. Gillis, and L. M. Lichtenstein. 1989. Recombinant IL-3 induces histamine release from human basophils. J. Immunol. 142:3527-3532.

17. Zachariae, C. O. C., A. O. Anderson, H. L. Thompson, E. Appella, A. Mantovani, J. O. Oppenheim, and K. Matsushima. 1990. Properties of monocyte chemotactic and activating factor (MCAF) purified from a human fibrosarcoma cell line. J. Exp. Med. 171:2177-2182.

18. Gallin, J. L., and D. G. Wright. 1974. Role of secretory events in modulating human neutrophil chemotaxis. J. Clin. Invest. 62:1364-1374.

19. MacDonald, S. M., J. M. White, A. Kagey-Sobotka, D. W. MacGlashan, Jr., D. Proud, and L. M. Lichtenstein. 1987. Heterogeneity of IgE: transfer of $\mathrm{D}_{2} \mathrm{O}$ sensitivity. Clin. Res. 35:641A. (abstr.).

20. Naclerio, R. M., D. Proud, A. Togias, N. F. Adkinson, Jr., D. A. Meyers, A. Kagey-Sobotka, M. Plaut, P. S. Norman, and L. M. Lichtenstein. 1985. Inflammatory mediators in late antigen-induced rhinitis. N. Engl. J. Med. 313:65-70.

21. Warner, J., M. Pienkowski, M. Plaut, P. S. Norman, and L. M. Lichtenstein. 1986. Identification of histamine releasing factor(s) in the late phase of cutaneous IgE-mediated reactions. J. Immunol. 136:2583-2590.

22. Alam, R., J. Welter, P. A. Forsythe, M. A. Lett-Brown, J. A. Rankin, M. Boyars, and J. A. Grant. 1990. Detection of histamine release inhibitory factorand histamine releasing factor-like activities in bronchoalveolar lavage fluids. Am. Rev. Respir. Dis. 141:666-671.

23. Alam, R., P. Kuna, J. Rozniecki, and B. Kuzminska. 1987. The magnitude of spontaneous production of histamine releasing factor (HRF) by lymphocytes in vitro highly correlates with state of bronchial hyperreactivity in patients with asthma. J. Allergy Clin. Immunol. 79:103-108.

24. Sampson, H. A., K. R. Broadbent, J. Bernhisel-Broadbent. 1989. Spontaneous basophil histamine release and histamine releasing factor in patients with atopic dermatitis and food hypersensitivity. N. Engl. J. Med. 321:228-232. 